\title{
Table of cases and legislation
}

\section{The Court of Justice}

Case 1/58 Stork [1959] ECR 0043 35

Joined Cases 36, 37, 38/59 and 40/59 Ruhrkohlen [1960] ECR 0857. .35

Joined Cases 5 to 11 and 13 to 15/62 San Michele [1962] ECR 0449 167,227

Case 25/62 Plaumann [1963] ECR 0095 129-131, 139-141

Case 26/62 Van Gend en Loos [1963] ECR 1 ...33

Case 32/62 Alvis [1963] ECR 0049 185

Case 6/64 Costa [1964] ECR 585 .33

Case 40/64 Sgarlata [1965] ECR 0259. 35,140

Case 6/68 Zuckerfabrick Watenstedt English special edition 0409 140

Case 11/70 Internationale Handelsgesellschaft [1970] ECR 1125 $7,10,16-17$ $38,65,99,200$

Case 22/70 Commission v Council [1971] ECR 0263. .30

Case 7/71 Commission v France [1971] ECR 1003 192

Case 7/72 Boehringer Mannheim [1972] ECR 1281 240

Case 4/73 Nold [1974] ECR 0491 $.8,35,63-64,250$

Case 17/74 Transocean Marine Paint [1974] ECR 1063 166

Case 36/75 Rutili [1975] ECR 1219 .46

Case 33/76 Rewe-Zentralfinanz [1976] ECR 1989 .............................133, 135-136

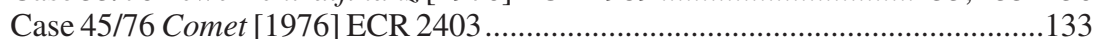

Case 85/76 Hoffman-La Roche [1979] ECR 0461 ...............................................166

Case 101/76 Scholten Honig [1977] ECR 0797 ....................................................140

Case 121/76 Alessandro Moli [1977] ECR 1971 ....................................................166

Case 120/78 Rewe-Zentral AGv Bundesmonopolverwaltung für Branntwein [1979] ECR 0649.

Joined Cases 209 to 215 and 218/78 van Landewyck [1980] ECR 3125........179-180

Case 44/79 Hauer [1979] ECR 3727 $8,10,35,65-66,250$

Case 98/79 Pecastaing [1980] ECR 0691 152

Case 136/79 National Panasonic [1980] ECR 2033 167,227

Case 155/79 AM \& $S$ [1982] ECR 1575 167,172

Joined Cases 100 to 103/80 Musique Diffusion française [1983] ECR 1825.166, 179 Case 283/81 CILFIT [1982] ECR 3415 ............................................................257

Case 322/81 Michelin [1983] ECR 3461 166

Case 14/83 Von Colson [1984] ECR 1891

Case 294/83 Les Verts [1986] ECR 1339

Case 152/84 Marshall [1986] ECR 0723

Case 222/84 Johnston [1986] ECR 1651.

Case 268/84 Valsabbia [1987] ECR 0353 $.53,133,135,153$ $29-30,127$ 
Joined Cases 133-136/85 Walter Rau [1987] ECR 2289...................................132

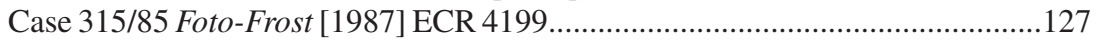

Case 12/86 Demirel [1987] ECR 3719 ..........................................................4

Case 222/86 Heylens [1987] ECR 4097 ....................................152-154, 158, 207

Case 249/86 Commission v Germany [1989] ECR 1263 ........................................96

Joined Cases 46/87 and 227/88 Hoechst [1989] ECR 2859..................166-168, 207,

226-227, 239

Case 229/87 Commission v Greece [1988] ECR 6347 ........................................189

Case 265/87 Schraeder [1989] ECR 2237 ..............................................63, 67

Case 374/87 Orkem [1989] ECR 3283 .......................167, 173-176, 228-231, 239

Order in Case C-2/88 IMM Zwartveld [1990] ECR I-3365 ...................................133

Case 5/88 Wachauf [1989] ECR 2609...........................................................45, 64

Case C-331/88 FEDESA [1990] ECR I-04023 .................................................62

Case C-213/89 Factortame [1990] ECR I-243 ….................................... 53, 135

Case C-260/89 ERT [1991] ECR I-2925 …..................................45, 47, 70, 77

Case C-309/89 Codorniu [1994] ECR I-1853 ..................................................130

Case C-340/89 Vlassopoulou [1991] ECR I-2357 .................................................154

Case C-358/89 Extramet [1991] ECR I-2501 .......................................................130

Case C-159/90 Grogan [1991] ECR I-4685 ................................69, 77, 222, 232

Joined Cases C-6/90 and C-9/90 Frankovich [1991] ECR I-5357 ..................53, 135

Joined Cases C-48/90 and C-66/90 The Netherlands and Others $v$ Commission

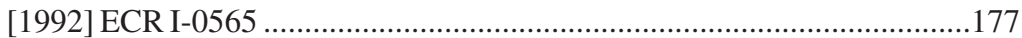

Joined Cases C-87/90 to C-89/90 Verholen [1991] ECR I-3757 ..................137, 153

Case C-177/90 Kühn [1990] ECR I-0035 ........................................................63

Case C-208/90 Emmott [1991] ECR I-4269 .....................................................138

Case C-97/91 Oleificio Borelli [1992] ECR I-6313 …...............................209-210

Case C-206/91 Koua Poirrez [1992] ECR I-6685 ......................................232-233

Case C-2/92 Bostock [1994] ECR I-0955 ...........................................................64

Case C-91/92 Dori [1994] ECR I-3325 ..............................................................53

Case C-135/92 Fiskano [1994] ECR I-2885 .......................................................185

Case C-199/92 Hüls [1999] ECR I-4287 ..........................................................23

Case C-23/93 TV 10 [1994] ECR I-4795 …....................................................... 71

Joined Cases C-46/93 and C-48/93 Factortame [1996] ECR I-1029 .....................53

Case C-280/93 Germany v Council [1994] ECR I-4973 ............................62-64, 67

Case C-306/93 Winzersekt [1994] ECR I-5555.......................................62-63, 66

Case C-312/93 Peterbroeck [1995] ECR I-4599 ..........................................134, 136

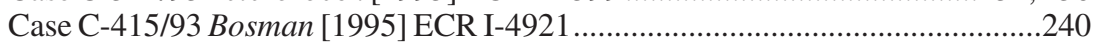

Case C-16/94 Dubois [1995] ECR I-2421 .........................................................64

Case C-22/94 IFA [1997] ECR I-1809 .......................................................63-66

Case C-36/94 Siesse [1995] ECR I-3573 .............................................................116

Case C-44/94 National Federation of Fishermen's Organization [1995] ECR I-3115. $.62-63$

Case C-158/94 Commission v Italy [1997] ECR I-5789 ......................................190

Case C-209/94 Buralux [1996] ECR I-0615 .......................................................132

Case C-32/95 P Lisrestal [1996] ECR I-5373 .....................................................185

Case C-185/95 P Baustahlgewebe [1998] ECR I-8417 ..............................183-184

Case C-299/95 Kremzow [1997] ECR I-2629 ................................................45, 47

Case C-321/95 P Greenpeace [1998] ECR I-1651 ...........................................132 
Case C-368/95 Familiapress [1997] ECR I-3689 $70,77,240,242$

Case C-48/96 P Windpark Groothusen [1998] ECR I-2873

Case C-157/96 NFU [1998] ECR I-2211 ............................................................62

Case C-200/96 Metronome Musik [1998] ECR I-1971 ..........................................60

Case C-207/96 Commission v Italy [1997] ECR I-6869 .......................................189

Case C-326/96 Levez [1998] ECR I-0783 ...........................................................156

Case C-328/96 Commission v Austria [1999] ECR I-7479 ..................................189

Case C-104/97 P Atlanta [1999] ECR I-6983 ....................................................65

Case C-7/98 Krombach [2000] ECR I-1935. $86-87,105,202$

Order in Case C-17/98 Emesa Sugar [2000] ECR I-0665 ........................222-225

Joined Cases C-174/98 P and C-189/98 P Van der Wal [2000] ECR I-0001 ........208, $210-212$

Case C-377/98 Netherlands v Parliament and Council [2001] ECR I-7079 ........121

Case C-443/98 Unilever [2000] ECR I-7535 .......................................................53

Case C-462/98 P Mediocurso [2000] ECR I-7183 ...........................................185

Case C-1/99 Kofisa Italia [2001] ECR I-0207 ..................................................154

Case C-41/99 P Sadam Zuccherifici [2001] ECR I-4239 ...................................131

Case C-159/99 Commission v France [2001] ECR I-4007 …..............................190

Case C-194/99 P Thyssen Stahl [2003] ECR I-10821 .......................................183

Case C-213/99 de Andrade [2000] ECR I-11083 ..............................................116

Case C-226/99 Siples [2001] ECR I-0277 ....................................................154

Joined Cases C-238/99 P, C-244/99 P, C-245/99 P, C-247/99 P, C-250/99 P, C-251/99 P, C-252/99 P, C-254/99 P Limburgse Vinyl [2002] ECR I-8375 $168,175,180,183,227-229$

Case C-274/99 Connolly [2001] ECR I-1611 $111-113,240$

Case C-315/99 P Ismeri Europa [2001] ECR I-5281 166

Case C-333/99 Commission v France [2001] ECR I-1025 ..................................190

Case C-353/99 Hautala [2001] ECR I-9565 ......................................................101

Case C-413/99 Baumbast [2002] ECR I-7091 ......................................................96

Case C-439/99 Commission v Italy [2002] ECR I-0305 ......................................191

Case C-453/99 Courage and Crehan [2001] ECR I-6297 ...................................156

Case C-459/99 MRAX [2002] ECR I-6591 .......................................................152

Case C-3/00 Denmark v Commission [2003] ECR I-2643 ..................................187

Joined Cases C-20/00 and C-64/00 Booker Aquaculture [2003] ECR I-7411 $.64,66$

Case C-50/00 P UPA [2002] ECR I-6677 ......................28, 126-131, 140-149, 206

Case C-60/00 Carpenter [2002] ECR I-6279 72,77

Case C-94/00 Roquette Frères [2002] ECR I-9011 ..........48, 77, 133, 168, 207, 227, 239,251

Case C-112/00 Schmidberger [2003] ECR I-5659 .............. 50, 74-75, 78, 201, 240,

Case C-185/00 Commission v Finland [2003] ECR I-14189 ..............................191

Case C-255/00 Grundig Italiana [2002] ECR I-8003 .................................137-138

Joined Cases C-465/00, C-138/01 and C-139/01 ORF [2003] ECR I-4989 ....79-80, $83,100-101,204-205,251$

Case C-13/01 Safalero [2003] ECR I-8679 137,156

Case C-101/01 Lindqvist [2003] ECR I-12971 .........45, 83-84, 98-99, 205, 240, 250

Case C-109/01 Akrich [2003] ECR I-9607 ........................................48, 72, 251

Case C-145/01 Commission v Italy [2003] ECR I-5581 190 
Joined Cases C-187/01 and C-385/01 Gözütok and Brügge [2003] ECR

$\mathrm{I}-1345$

Case C-245/01 RTL [2003] ECR I-12491 68,72

Case C-276/01 Steffensen [2003] ECR I-3735 $42,50,135,159,185,207$

Joined Cases C-397/01 to C-403/01 Pfeiffer [2004] ECR I-8835 .............................34

Case C-467/01 Eribrand [2003] ECR I-6471 ......................................................155

Joined Cases C-482/01 and C-193/01 Orfanopoulos [2004] ECR I-5257 .......72, 77,

Case C-491/01 BAT [2002] ECR I-11453

$62,147-148,206$

Case C-494/01 Commission v Ireland [2005] ECR I-3331 190-191

Joined Cases C-37/02 and C-38/02 Di Lenardo [2004] ECR I-6911 $63-65,67$

Case C-36/02 Omega [2004] ECR I-9609 $.50,75-76,78,201$

Joined Cases C-65/02 P and C-73/02 P Thys

Case C-71/02 Karner [2004] ECR I-3025 229

Case C-116/02 Gasser [2003] ECR I-14696 $42,71-72,77,201$

Case C-167/02 P Willi Rothley [2004] ECR I-3149 104

Case C-174/02 Streekgewest [2005] ECR I-0085 140,149

Joined Cases C-189/02 P, C-202/02 P, C-205/02 P to C-208/02 P and C-213/02 P Dansk Rørindustri [2005] ECR I-5425 $178,239-240$

Case C-263/02 P Commission v Jégo-Quéré [2004] ECR I-3425 .......140, 142-145, $148-149$

Case C-287/02 Spain v Commission [2005] ECR I-5093 162,187

Joined Cases C-387/02, C-391/02 and C-403/02 Criminal proceedings against Berlusconi [2005] ECR I-3565 .239

Case C-304/02 Commission v France [2005] ECR I-0626 ............189-191, 193-195

Case C-416/02 Commission v Spain [2005] ECR I-7487 190

Case C-441/02 Commission v Germany [2006] ECR I-3449 .72

Case C-459/02 Willy Gerekens [2004] ECR I-7315 .239

Case C-131/03 P R.J. Reynolds [2006] ECR I-7795 $127,140,148$

Case C-160/03 Spain v Eurojust [2005] ECR I-2077 147

Case C-177/03 Commission v France [2004] ECR I-11671 ................................192

Case C-210/03 Swedish Match [2004] ECR I-11893 ...........................................64

Case C-291/03 MyTravel [2005] ECR I-8477 …................................................137

Case C-295/03 P Alessandrini [2005] I-5673 ..................................................67

Case C-347/03 Regione Autonoma Friuli-Venezia-Giulia [2005] ECR I-3785 ....65, 67,241

Case C-380/03 Germany v Parliament and Council [2006] ECR I-11573 .......62, 72

Case C-397/03 P Archer Daniels Midland [2006] ECR I-4429 ............................178

Case C-461/03 Gaston Schul [2005] ECR I-10513 ...............................................127

Case C-469/03 Miraglia [2005] ECR I-2009 ...................................................117

Case C-511/03 Ten Kate [2005] ECR I-8979 .....................................................132

Case C-540/03 Parliament v Council [2006] ECR I-5769.............. 95-97, 250

Case C-33/04 Commission v Luxembourg [2005] ECR I-10629 ...........189-190, 192

Case C-144/04 Mangold [2005] ECR I-9981 ....................................11, 33-64, 53

Case C-145/04 Spain v UK [2006] ECR I-7917 $208,234,240$

Joined Cases C-154/04 and C-155/04 Alliance for Natural Health [2005] ECR I-6451 64, 241, 250-251

Joined Cases C-295/04 to C-298/04 Manfredi and Others [2006] ECR I-6619 ....156 
Case C-300/04 Eman and Sevinger [2006] ECR I-8055 ...

$233-234,240$

Case C-308/04 P SGL Carbon [2006] ECR I-5977

174,240

Case C-328/04 Vajnai [2005] ECR I-8577

Case C-341/04 Eurofood [2006] ECR I-3813

$87,106,202$

Case C-354/04 P Gestoras [2007] ECR I-1579 28-30, 34, 45, 55, 147, 206

Case C-355/04 P Segi [2007] ECR I-1657 28-30, 34, 45, 55, 147, 206

Joined Cases C-403/04 P and C-405/04 P Sumitomo [2007] ECR I-0729 .... 183

Case C-407/04 P Dalmine [2007] ECR I-0829 174, 228-229, 239

Case C-411/04 P Salzgitter Mannesmann [2007] ECR I-0959 ............................165

Case C-436/04 Van Esbroeck [2006] ECR I-2333 .............................................117

Case C-438/04 Mobistar [2006] ECR I-6675 114

Case C-479/04 Laserdisken [2006] ECR I-8089 68,250

Case C-523/04 Commission v the Netherlands [2007] ECR I-3267 .....................192

Case C-35/05 Reemtsma Cigarettenfabriken [2007] ECR I-2425 ........................137

Joined Cases C-39/05 P and C-52/05 P Turco [2008] ECR I-04723 .......................84

Case C-64/05 P Sweden v Commission [2007] ECR I-11389 ..............................212

Case C-150/05 van Straaten [2006] ECR I-9327 .................................................117

Joined Cases C-222/05 to C-225/05 van der Weerd [2007] ECR I-4233 ................136

Case C-229/05 P Ocalan and Vanly [2007] ECR I-0439 ....................................244

Case C-260/05 P Sniace [2007] ECR I-10005 ..................................................140

Case C-266/05 P Sison [2007] ECR I-1233 ...................................................101

Case C-283/05 ASML [2006] ECR I-12041 ..........................86, 88, 107-108, 202

Case C-303/05 Advocaten voor de Wereld [2007] ECR I-3633 ...29, 45, 89, 114, 204

Case C-305/05 Ordre des barreux francophones [2007] ECR I-5305 ...........45, 111

Case C-306/05 Rafael Hoteles [2006] ECR I-11519 ..........................................244

Case C-341/05 Laval Partneri [2007] ECR I-11767 ..............................75-76, 78

Case C-370/05 Festersen [2007] ECR I-1144 .............................................70-71

Joined Cases C-402/05 P and C-415/05 P Kadi [2008] ECR I-6351 .............7-9, 13, 29-30, 35, 37-38, 45, 163, 200

Case C-432/05 Unibet [2007] ECR I-2271 ..................129, 134-136, 155, 206-207

Case C-438/05 ITWF [2007] ECR I-10779 ..................................................75-76

Case C-2/06 Kempter [2008] ECR I-0411 .............................................136, 138

Case C-3/06 P Groupe Danone [2007] ECR I-1331 ....................178, 180, 239-240

Case C-105/06 Pupino [2005] ECR I-5285 .........................................34, 53, 242

Case C-215/06 Commission v Ireland [2008] ECR I-4911 ..................................138

Case C-244/06 Dynamic Medien [2008] ECR I-0505 ........................50, 75-78, 201

Case C-250/06 United Pan-Europe Communications [2007] ECR I-11135 .....71, 77

Case C-268/06 Impact [2008] ECR I-2483 ......................................................133

Case C-275/06 Promusicae [2008] ECR I-0271 .......................................78-79, 204

Case C-301/06 Ireland $v$ Parliament and Council [2009] ECR I-0593 ...................83

Case C-308/06 Intertanko [2008] ECR I-4057 ...........................................116-117

Joined Cases C-341/06 P and C-342/06 P Chronopost [2008] ECR I-4777 .....................................................................225-226, 243

Joined Cases C-399/06 P and C-403/06 P Hassan [2009] ECR I-11393 ..............200

Case C-442/06 Commission v Italy [2008] ECR I-2413 .......................................189

Case C-450/06 Varec [2008] ECR I-0581 .......................................111, 113, 238

Case C-521/06 P Athinaïki Techniki AE [2008] ECR I-5829 ..............................127

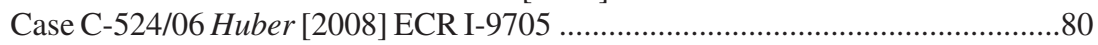


Case C-14/07 Weiss [2008] ECR I-3367

$87,108,202$

Case C-47/07 P Masdar [2008] ECR I-9761 $150-151$

Case C-73/07 Satamedia [2008] ECR I-9831 98-99, 205

Joined Cases C-125/07 P, C-133/07 P, C-135/07 P and C-137/07P Erste Group

Bank [2009] ECR I-8681

Case C-336/07 Kabel Deutschland [2008] I-10889

Case C-349/07 Sopropé [2008] ECR I-10369 $162,185-186,205$

Case C-369/07 Commission v Greece [2009] ECR I-5703 191, 193-194

Case C-385/07 P Der Grüne Punkt [2009] ECR I-6155 183-184

Case C-394/07 Gambazzi [2009] ECR I-2563 $87,106-107,202$

Case C-404/07 Katz [2008] ECR I-7607 242

Case C-420/07 Apostolides [2009] ECR I-3571 87,105

Case C-444/07 MG Probud [2010] ECR I-0417 .87

Case C-465/07 Elgafaji [2009] ECR I-0921 45,237

Case C-467/07 Gasparini [2008] ECR I-0040 90,117

Case C-477/07 P Agrofert [2008] ECR I-0105

Case C-491/07 Turanský [2008] ECR I-11039 84, 101-102

Joined Cases C-514/07 P, C-528/07 P and C-532/07 P Association de la presse internationale ASBL (API) [2010] ECR I-8533 $84,101,103-104,187$

Case C-518/07 Commission v Germany [2010] ECR I-1885

Case C-546/07 Commission v Germany [2010] ECR I-0439 241

Case C-550/07 P Akzo Nobel [2010] ECR I-8301 192

Case C-553/07 Rijkeboer [2009] ECR I-3889 $166,172-173$

Case C-555/07 Kücükdeveci [2010] ECR I-0365 100,205

Case C-568/07 Commission v Greece [2009] ECR I-4505

$11,33-34,53$

Case C-12/08 Mono Car Styling [2009] ECR I-6653 194

Case C-19/08 Petrosian [2009] ECR I-0495 137,155

Case C-28/08 P Bavarian Lager [2010] ECR I-6055 ........84-86, 102-103, 238, 241

Case C-40/08 Asturcom [2009] ECR I-9579 134

Case C-45/08 Spector Photo [2009] ECR I-12073 .243

Case C-63/08 Pontin [2009] ECR I-10467 134-135, 137, 206

Case C-66/08 Kozłowski [2008] ECR I-6041 .89

Case C-89/08 Commission v Ireland and Others [2009] ECR I-11245 .......166-167,

187,223

Case C-109/08 Commission v Greece [2009] ECR I-4657 193-194

Case C-127/08 Metock [2008] ECR I-6241 48,72

Case C-141/08 P Foshan Shunde Yongjian Housewares \& Hardware [2009] ECR I-9147 185

Case C-265/08 Federutility [2010] ECR I-3377 .28 Joined Cases C-317/08 to C-320/08 Alassini [2010] ECR I-2213 ........137, 158, 241 Case C-350/08 Commission v Lithuania [2010] ECR I-10525 ............................192

Case C-423/08 Commission v Italy [2010] ECR I-5449 ......................................185

Case C-439/08 VEBIC [2010] ECR I-12471 .......................................................154

Case C-578/08 Chakroun [2010] ECR I-1839 ...........................................95, 241

Case C-27/09 P France v People's Mojahedin Organization of Iran nyr. .....162-163

Case C-31/09 Bolbol [2010] ECR I-5539 .90

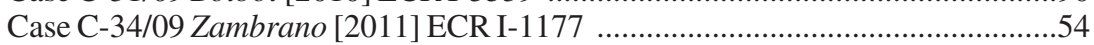

Joined Cases C-92/09 and C-93/09 Schecke [2010] ECR I-11063 ......................243 
Case C-145/09 Tsakouridis [2010] ECR I-11979

$72,77,242$

Case C-196/09 Paul Miles [2011] ECR I-5105 146

Case C-197/09 R-XII M. v European Medicines Agency [2009] ECR I-12033 ....187

Case C-208/09 Sayn-Wittgenstein [2010] ECR I-13693 .......................................78

Case C-235/09 DHL Express France [2011] ECR I-2801 ....................................152

Case C-240/09 Lesoochranárske zoskupenie VLK [2011] ECR I-1255 152

Case C-246/09 Bulicke [2010] ECR I-7003 $136-137$

Case C-272/09 P KME nyr. $41,177-178,184-185$

Case C-279/09 DEB [2010] ECR I-13849 .........36, 55, 150, 158-159, 207, 242-243

Case C-336/09 P Poland v Commission nyr. 29,55

Case C-403/09 PPU Detiček [2009] ECR I-12193

Order in Case C-457/09 Chartry [2011] ECR I-0819 ........................................48

Case C-543/09 Deutsche Telekom [2011] ECR I-3441 ......................................98

Case C-546/09 Aurubis Balgaria [2011] ECR I-2531 ......................................116

Case C-548/09 P Bank Melli Iran nyr. .................................................................200

Case C-550/09 Criminal proceedings against $E$ and $F$ [2010] ECR I-6213

Case C-34/10 Brüstle, nyr.

$29,144,148$

Case C-70/10 Scarlet Extended nyr.

$.79,204$

Order in Case C-73/10 P IFG Weichert [2010] ECR I-11535 .....................225, 243

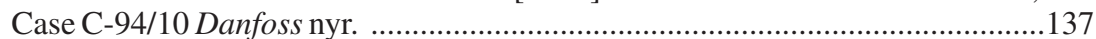

Case C-242/10 ENEL Produzione nyr. .............................................................28

Case C-386/10 P Chalkor nyr. .................................40-41, 178, 180, 184-185, 245

Case C-400/10 PPU McB [2010] ECR I-8965 …................................36, 109, 244

Case C-405/10 Garenfeld nyr. .............................................................116, 205

Joined Cases C-411/10 and C-493/10 N.S. nyr. ....36, 45-46, 48, 50, 90-91, 118-119,

208,234

Case C-416/10 Križan nyr. ...........................................68, 133, 137-138, 152, 206

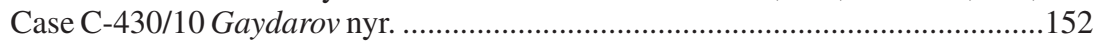

Case C-461/10 Bonnier Audio nyr ............................................................79, 204.

Joined Cases C-468/10 and C-469/10 ASNEF nyr. ..............................99-100, 205

Case C-491/10 PPU Pelz [2010] ECR I-14247 ...........................................110, 202

Case C-507/10 Criminal Proceedings against $X$ nyr. ..........................................242

Case C-571/10 Kamberaj nyr. ...........................................................................48

Case C-610/10 Commission v Spain nyr. ...................................................194-195

Case C-617/10 Fransson nyr. .............................................................47-49, 217

Case C-619/10 Trade Agency nyr. ........................................87-88, 105-107, 202

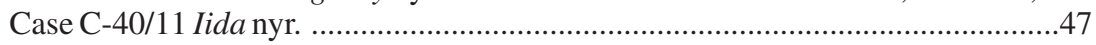

Case C-42/11 João Pedro Lopes Da Silva Jorge nyr. .......................................34, 53

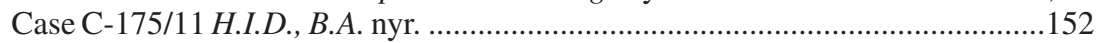

Case C-199/11 Europese Gemeenschap nyr. .........40, 126, 156-157, 184-185, 207 ,

Case C-244/11 Commission v Greece nyr. ........................................................28

Case C-256/11 Dereci nyr. $47,126,245$

Case C-277/11 M.M. nyr. .....................................................162, 185-186, 205

Case C-279/11 Commission v Ireland nyr. ........................................................195

Case C-283/11 Sky Österreich nyr. ....................................40, 63, 71, 101, 245 
Joined Cases C-356/11 and C-357/11 O. and S. nyr.

Case C-396/11 Radu nyr.

Case C-399/11 Melloni nyr. $38,49-50,115$

Order in Case C-466/11 Currà and Others nyr. 48

Case C-293/12 Digital Rights Ireland, OJ 2012 C 258/11 . .....................................83

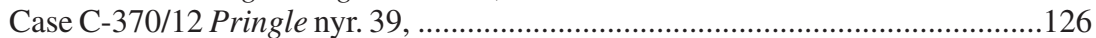

Opinion 1/91 The EEA Agreement [1991] ECR 6079 .28

Opinion 2/94 Accession by the Community to the European Convention for the Protection of Human Rights and Fundamental Freedoms [1996] ECR I-1759 $.7,8,35$

Opinion 1/09 The draft agreement on the European and Community Patents Court [2011] ECR I-1137 .127, 132

Opinion of Advocate General Capotorti in Case 98/79 Pecastaing [1980] ECR 0691

Opinion of Advocate General Mancini in Case 294/83 Les Verts [1986] ECR 1339

Opinion of Advocate General Jacobs in Case C-168/91 Konstantinidis [1993] ECR I-1191

Opinion of Advocate General Colomer in Case C-387/97 Commission v Greece [2000] ECR I-5047

Opinion of Advocate General Jacobs in Case C-50/00 P UPA [2002] ECR I-66779

Opinion of Advocate General Maduro in Case C-380/05 Centro Europa 7 [2005] ECR I-0349 $4,50-51$

Opinion of Advocate General Maduro in Case C-402/05 P Kadi [2008] ECR I-6351

Opinion of Advocate General Kokott in Case C-526/08 Commission v Luxembourg [2010] ECR I-6151

Opinion of Advocate General Sharpston in C-272/09 P KME nyr. 182

\section{The General Court (Former Court of First Instance)}

Case T-114/92 BEMIM [1995] ECR II-0147 .....................................................176

Case T-34/93 Société Générale [1995] ECR II-0545 ..........................................220

Case T-346/94 France-aviation [1995] ECR II-2841 ................................208, 210

Case T-174/95 Svenska Journalistförbundet [1998] ECR II-2289 .......................101

Case T-42/96 Eyckeler\&Malt [1998] ECR II-0401 ............................................210

Case T-124/96 Interporc [1998] ECR II-0231 .................................................101

Case T-135/96 UEAPME [1998] ECR II-2335 ................................................130

Case T-83/98 Van der Wal [1998] ECR II-0545 ...............................................211

Case T-112/98 Mannesmannröhren-werke [2001] ECR II-0729 ...174, 230-231, 239

Joined Cases T-172 and T-175-177/98 Salamander [2000] ECR II-2487 .............132

Case T-191/99 Petrie [2001] ECR II-3677 .........................................................191

Case T-211/00 Kuijer [2002] ECR II-0485 .....................................................102

Case T-177/01 Jégo-Quéré [2002] ECR II-2365 ................................130, 142-144

Case T-306/01 Yusuf [2005] ECR II-3533 …................................................200

Joined Cases T-22/02 and T-23/02 Sumitomo [2005] ECR II-4065 ......................239

Case T-38/02 Groupe Danone [2005] ECR II-4407 166,176 
Case T-47/02 Danzer [2006] ECR II-1779

239,251

Case T-59/02 Archer Daniels Midland [2006] ECR II-3627

Case T-228/02 Organisation des Modjahedines du peuple d'Iran [2006] ECR

II-4665

Case T-292/02 Confservizi [2009] ECR II-1659

140

Case T-309/02 Acegas-APS [2009] ECR II-1809 ...................................................140

Case T-2/03 Verein für Konsumenteninformation [2005] ECR II-1121 ................102

Case T-47/03 Sison [2007] ECR II-0073 ............................................................24

Order in Case T-196/03 EFfCI [2004] ECR II-4263 ............................................143

Case T-194/04 Bavarian Lager [2007] ECR II-4523 ................................103, 238

Case T-21/05 Chalkor [2010] ECR II-1895 .................................................178

Case T-138/07 Schindler Holding [2011] ECR II-4819 ............................166, 180

Case T-385/07 FIFA [2011] ECR II-0205 7 ..........................................3-74, 242

Case T-55/08 UEFA [2011] ECR II-0271 ….....................................73-74, 242

Case T-62/08 ThyssenKrupp Terni [2010] ECR II-3229 ....................................244

Case T-68/08 FIFA [2011] ECR II-0349 ..............................................73-74, 242

Case T-82/09 Dennekamp [2011] ECR II-0418 ..................................................103

Case T-85/09 Kadi [2010] ECR II-5177 ................................................200, 244

Case T-300/10 Internationaler Hilfsfonds nyr. ...............................................103

\section{The Civil Service Tribunal}

Case F-80/08 Wenig [2009] ECR FP-I-A-1-0479, FP-II-A-1-2609 ............239, 243

Case F-50/09 DEP di Lusignano nyr. .............................................................225

Case F-103/09 John Allen nyr. ......................................................................24

The European Court of Human Rights (and Former European Commission of Human Rights)

Commission Decision of 6 February 1967 X. v Iceland D.R. ...................5, 86, 238

Belgian Linguistics Case (No 2) Series A no 6 (1968) ..........................................248

Handyside v UK Series A no 24 (1976) .......................................................247-249

Commission Report of 12 July 1977 Brüggeman and Scheuten v Germany D.R 10,137238

Commission Decision of 10 July 1978 in Confédération française démocratique du travail v the European Communities, alternatively: their Member States a) jointly and $b$ ) severally D.R.

Sunday Times $v$ UK Series A no 30 (1979) 13,235235

Sporrong and Lönnroth $v$ Sweden Series A no 52 (1982) ..........................250-251

Commission Decision of 9 December 1987 Etienne Tęte v France D.R. ..54, 62, 235

Leander v Sweden Series A no 116 (1987) .........................................................250

Bricmont v Belgium Series A no 158 (1989) ......................................................252

Commission Decision of 11 July 1989 Société Stenuit v France D.R. ....61, 131, 180

Commission Decision of 9 February 1990 M. \& Co. v Germany D.R. ..........64, 138,

235-236, 253-254

Report of the Commission of 30 May 1991 Société Stenuit v France Series A no 232-A (1991) 
Open Door Counselling and Dublin Well Woman v Ireland Series A no 246 (1992)

Niemietz v Germany Series A no 251-B (1992) 226-227, 238, 251

Funke and others $v$ France Series A no 256-A (1993) .......................................229

Cantoni v France ECHR 1996-V ......................................................................2252

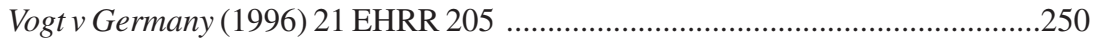

Saunders v UK (1997) 23 EHRR 313 .............................................228-231, 252

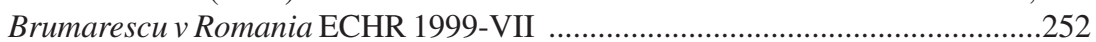

Grigoriades v Greece (1999) 27 EHRR 464 .......................................................250

Waite and Kennedy v Germany (1999) 30 EHRR ......................................261 253

Matthews v UK (1999) 30 EHRR 361 ..............................233-234, 236, 240, 254

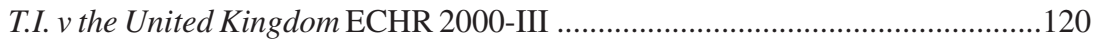

Rotaru v Romania ECHR 2000-V .....................................................................228

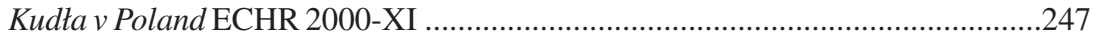

Heaney and McGuiness v Ireland ECHR 2000-XII ...........................................231

Amman v Switzerland (2000) 30 EHRR 843 ......................................................238

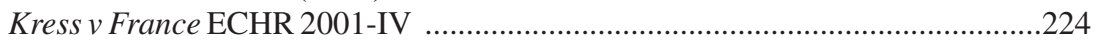

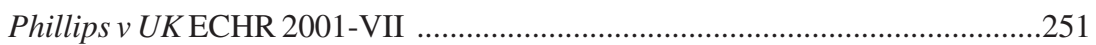

Boultifv Switzerland (2001) 33 EHRR 1179 ..........................................72, 251

Pretty v UK ECHR 2002-III .............................................................238-239

Janosevic v Sweden ECHR 2002-VII .....................................................180, 251

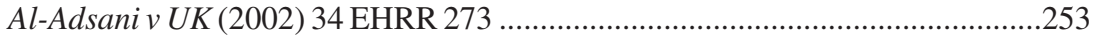

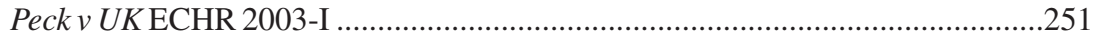

Koua Poirrez v France ECHR 2003-X ..................................................232-233

Decision of 3 June 2004 OOO Neste St. Petersburg and Others v Russia (App. 69042/01, 69050/01, 69054/01, 69055/01, 69056/01, 69058/01, not reported)

Jahn and others $v$ Germany ECHR 2005-IV ..................................................250

Bosphorus v Ireland ECHR 2005-VI .....7, 15, 21, 128-129, 208, 217, 224, 236, 245 ,

$247,429,252-256,258,261$

Decision of 13 January 2005 in EMESA SUGAR NV v the Netherlands (App. 62023/

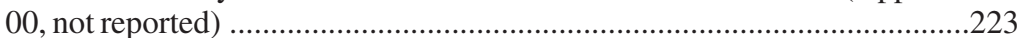

Achour v France (2005) 41 EHRR 36 .........................................................252

Sejdovic v Italy ECHR 2006-II .............................................................251-252

Valico S.r.l. v Italy ECHR 2006-III ...........................................................181-182

Jalloh v Germany ECHR 2006-IX .................................................................22

Hermi v Italy ECHR 2006-XII ......................................................................252

Jussila v Finland ECHR 2006-XIII ..............................................................180

Decision of 2 December 2008 K.R.S. v the United Kingdom (App. 32733/08, not

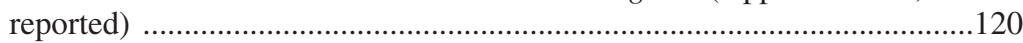

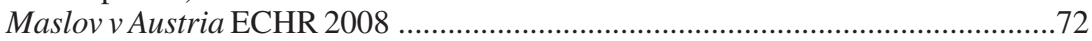

Cooperative Producentenorganisatie van de Nederlandse Kokkelvisserij U.A. v the Netherlands ECHR 2009 205, 208, 224-225, 254, 256 M.S.S. v Belgium and Greece ECHR 2011 ..............................119-120, 234, 254 Karoussiotis v Portugal ECHR 2011 ............................................................189 Judgment of 20 September 2011 Ullens de Schooten and Rezabek v Belgium (App. No. 3989/07 and 38353/07, not reported) 
Judgment of 27 September 2011 Menarini Diagnostics v Italy (App. 43509/08, not reported)

Decision of 27 September 2011 Pietro Pianese $v$ Italy and the Netherlands (App. $14929 / 08$, not reported)

Decision of 3 April 2012 in Lechouritou v Germany and the 26 other Member States of the European Union (App. 37937/07, not reported)

Michaudv France ECHR 2012

$112,128-129,208,253-256$

Segame v France ECHR 2012

\section{Other Jurisdictions}

Germany: BVerfGE 37, 271 (1974) (So lange I), $.280,66$

Germany: BVerfGE 73, 339 (1986) (So lange II)

\section{EU Legislation and Documents}

Regulation No 17: First Regulation implementing Articles 85 and 86 of the Treaty, OJ 1962 L 13/204 $163,166-167$

Directive 64/221/EEC of 25 February 1964 on the co-ordination of special measures concerning the movement and residence of foreign nationals which are justified on grounds of public policy, public security or public health, OJ 1964 L 56/850

Regulation 259/68/EEC, Euratom, ECSC of 29 February 1968 laying down the Staff Regulations of Officials and the Conditions of Employment of Other Servants of the European Communities and instituting special measures temporarily applicable to officials of the Commission, OJ 1968 L 56/1

Regulation 1612/68/EEC of 15 October 1968 on freedom of movement for workers within the Community, OJ 1968 L 257/2

Directive 76/207/EEC of 9 February 1976 on the implementation of the principle of equal treatment for men and women as regards access to employment, vocational training and promotion, and working conditions, OJ $1976 \mathrm{~L}$ $39 / 40$

Joint Declaration by the European Parliament, the Council and the Commission concerning the Protection of Fundamental Rights and the European Convention for the Protection of Human Rights and Fundamental Freedoms, OJ 1977 C 103/1

Directive 79/7/EEC of 19 December 1978 on the progressive implementation of the principle of equal treatment for men and women in matters of social security, OJ 1979 L 6/24

Commission White Paper on Completing the Internal Market, COM (1985) 310 final

Directive 89/552/EEC of 3 October 1989 on the coordination of certain provisions laid down by Law, Regulation or Administrative Action in Member States concerning the pursuit of television broadcasting activities, OJ 1989 L 298/23 ... 
Directive 89/665/EEC of 21 December 1989 on the coordination of the laws, regulations and administrative provisions relating to the application of review procedures to the award of public supply and public works contracts, OJ 1989 L 395/33, as amended by Directive 92/50/EEC of 18 June 1992 relating to the coordination of procedures for the award of public service contracts, OJ 1992 L 209/1 .113

Directive 91/308/EEC of 10 June 1991 on prevention of the use of the financial

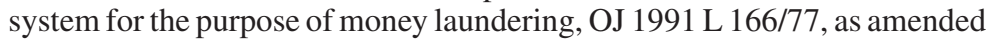
by Directive 2001/97/EC of 4 December 2001, OJ 2001 L 344/76 ..............111

Declaration annexed to the EU Treaty on the right of access to information, OJ 1992 C 191

Regulation 2913/92/EEC of 12 October 1992 establishing the Community Customs Code, OJ 1992 L 302/1, as amended by Council Regulation (EC) 1791/2006 of 20 November 2006, OJ 2006 L 363/1

Directive 93/36/EEC of 14 June 1993 coordinating procedures for the award of public supply contracts, OJ 1993 L 199/1

Regulation 2454/93/EEC of 2 July 1993 laying down provisions for the implementation of Council Regulation (EEC) No 2913/92 establishing the Community Customs Code, OJ 1993 L 253/1

Council Decision 93/731/EC of 20 December 1993 on public access to Council documents, OJ 1993 L 340/43 $.85,101,198$

Commission Communication to the Council, the Parliament and the Economic and Social Committee on public access to the institutions' documents, OJ 1993 C $156 / 5$

Commission Communication to the Council, the Parliament and the Economic and Social Committee on openness in the Community, OJ 1993 C 166/4 ..........101

Commission Decision 94/90 EC, ECSC, Euratom of 8 February 1994 on public access to Commission documents, OJ 1994 L 46/58 ..........................298, 211

Directive 95/46/EC of 24 October 1995 on the protection of individuals with regard to the processing of personal data and on the free movement of such data, OJ 1995 L 281/31 79, 83, 98-100, 205

Commission Memorandum on Applying Article 171 of the EC Treaty, OJ 1996 C $242 / 6$.

Directive 96/61/EC of 24 September 1996 concerning integrated pollution prevention and control, OJ 1996 L 257/26....

Parliament Decision 97/632/EC, ECSC, Euratom of 10 July 1997 on public access to European Parliament documents, OJ 1997 L 263/27

Convention on the service in the Member States of the European Union of judicial and extrajudicial documents in civil or commercial matters, drawn up by an act of the Council of the European Union dated 26 May 1997, OJ 1997 C 261/1

Directive 98/44/EC of 6 July 1998 on the legal protection of biotechnological inventions, OJ 1998 L 213/13 $.91,120,203$

Presidency Conclusions, Cologne European Council (1999), Annex IV <http:// www.consilium.europa.eu/ueDocs/cms_Data/docs/pressData/en/ec/ kolnen.htm > accessed 5 May 2013.

Regulation 1257/1999/EC of 17 May 1999 on support for rural development from the EAGGF, OJ 1999 L 160/80 197-198 
Convention implementing the Schengen Agreement of 14 June 1985, OJ 2000 L $239 / 19$

Regulation 1346/2000/EC of 29 May 2000 on insolvency proceedings, OJ $2000 \mathrm{~L}$ $160 / 1$ 87,106

Regulation 1348/2000/EC on the service of judicial and extrajudicial documents in civil and commercial matter and the objective of improving and expediting the cross-border transmission of documents, OJ 2000 L 160/37 $87,108-109$

Directive 2001/29/EC of 22 May 2001 on the harmonisation of certain aspects of copyright and related rights in the information society, OJ $2001 \mathrm{~L} 167 / 10$....68

Regulation 44/2001/EC of 22 December 2000 on jurisdiction and the recognition and enforcement of judgments in civil and commercial matters, OJ $2001 \mathrm{~L}$ $12 / 1$ ..86-88, 104-108, 202

Regulation 45/2001/EC of 18 December 2000 on the protection of individuals with regard to the processing of personal data by the Community institutions and bodies and on the free movement of such data, OJ 2000 L 8/1 .......85, 102-103

Framework Decision 2001/220/JHA of 15 March 2001 on the standing of victims in criminal proceedings, OJ 2001 L 82/1. Now replaced by Directive 2012/29/EU of 25 October 2012 establishing minimum standards on the rights, support and protection of victims of crime, OJ 2012 L 315/57

Regulation 1049/2001/EC of 30 May 2001 regarding public access to European Parliament, Council and Commission documents, OJ 2001 L 154/43 ....84-85, $101-103,198,212$

Directive 2002/21/EC of 7 March 2002 on a common regulatory framework for electronic communications networks and services, OJ $2002 \mathrm{~L}$ $108 / 33$

Directive 2002/58/EC of 12 July 2002 concerning the processing of personal data and the protection of privacy in the electronic communications sector, OJ 2002 L 201/37

Framework Decision 2002/584/JHA of 13 June 2002 on the European arrest warrant and the surrender procedures between Member States, OJ $2002 \mathrm{~L}$ $190 / 1$

Commission Communication on the Better Monitoring of the Application of Community Law, COM (2002) 725/4 final 188,191

Regulation 1/2003/EC of 16 December 2002 on the implementation of the rules on competition laid down in Articles 81 and 82 of the Treaty, OJ 2003 $\mathrm{L} 1 / 1$ 156-157, 163-165, 173, 175, 177-178, 180, 197, 227

Directive 2003/86/EC of 22 September 2003 on the right to family reunification, OJ 2003 L 251/12 $.94-95$

Regulation 343/2003/EC of 18 February 2003 establishing the criteria and mechanisms for determining the Member State responsible for examining an asylum application lodged in one of the Member States by a third country national, OJ 2003 L 50/1 $.91,118-119$

Regulation No 2201/2003/EC of 27 November 2003 concerning jurisdiction and the recognition and enforcement of judgments in matrimonial matters and the matters of parental responsibility, repealing Regulation (EC) No 1347/2000, OJ 2003 L 338/1 .88, 109-110 
Directive 2004/38/EC of 29 April 2004 on the right of citizens of the Union and their family members to move and reside freely within the territory of the Member States, OJ 2004 L 158/77 ....

Directive 2004/48/EC of 29 April 2004 on the enforcement of intellectual property rights, OJ $2004 \mathrm{~L} 157 / 45$ 133-134

Commission Regulation 773/2004/EC of 7 April 2004 relating to the conduct of proceedings by the Commission pursuant to Articles 81 and 82 of the EC Treaty, OJ 2004 L 123/18 $164-165$

Directive 2005/35/EC of 7 September 2005 on ship-source pollution and on the introduction of penalties for infringements, OJ $2005 \mathrm{~L}$ 255/11 .................116

Directive 2005/85/EC of 1 December 2005 on minimum standards of procedures in Member States for granting and withdrawing refugee status, OJ 2005 L 326/13 $133-134$

Directive 2006/24/EC of 15 March 2006 on the retention of data generated or processed in connection with the provision of publicly available electronic communications services or of public communications networks, OJ 2006 L $105 / 54$

Regulation 1013/2006/EC of 14 June 2006 on shipments of waste, OJ 2006 L 190/1

Guidelines on the method of setting fines imposed pursuant to Article 23(2)(a) of Regulation No 1/2003, OJ 2006 C 210/2 $177-178$

Regulation 42/2009/EC of 20 January 2009 setting up a Community regime for the control of exports, transfer, brokering and transit of dual-use items, OJ 2009 L $134 / 1$

Communication from the Commission to the European Parliament and the CouncilReport on the functioning of Regulation 1/2003, COM (2009) 206 final

Commission Recommendation 2009/524/EC on Measures to Improve the Functioning of the Single Market, OJ 2009 L 176/17

Regulation 1151/2012/EU of 21 November 2012 on quality schemes for agricultural products and foodstuffs, OJ 2012 L 343/1 198

\section{European Patent Organisation Decisions and Documents}

Rules 26-29 (ex Rules 23b-e) of the Implementing Regulations to the Convention on the Grant of European Patents of 5 October $1973<\mathrm{http}: / / w w w . e p o . o r g /$ law-practice/legal-texts/html/epc/2010/e/ma2.html> accessed 11 August 2013.

Decision of 25 November 2008, G 2/06, Official Journal EPO, May 2009, 306

\section{International Treaties}

Brussels Convention of 27 September 1968 on jurisdiction and the enforcement of judgments in civil and commercial matters, 1262 UNTS 153 .86-87, $104-105,223$

Oviedo Convention on Human Rights and Biomedicine and its Protocols,

CETS 164 and 186 92,123 
Marton Varju - 9781781951743 Downloaded from PubFactory at 04/26/2023 02:30:54PM via free access 